\title{
Treatment of obstructive epiphora in adults by balloon dacryocystoplasty
}

\author{
Zeynep Yazici, Bulent Yazici, Mufit Parlak, Haluk Erturk, Gursel Savci
}

\begin{abstract}
Aims-To determine the efficacy of dacryocystoplasty with balloon dilatation in the treatment of complete and partial obstruction of the lacrimal drainage system.

Methods-The procedure was performed on 26 patients with epiphora due to complete $(n=16)$ or partial $(n=10)$ obstruction of the lacrimal drainage system. A flexible tipped guide wire was introduced through the superior canaliculus into the inferior meatus and manipulated out of the nasal cavity. A $3 \mathrm{~mm}$ balloon was then introduced in a retrograde direction over the guide wire and dilated at the obstruction site.
\end{abstract}

Results-The procedure was technically successful in all patients with partial obstruction, but unsuccessful in four of 16 cases with complete obstruction. Reobstruction occurred in eight of 12 patients with complete obstruction, and in five of 10 patients with partial obstruction. The overall success rate was $25 \%$ for complete and $50 \%$ for partial obstructions. The mean follow up was 14 months (8-37 months).

Conclusion-Although the balloon dacryocystoplasty is a simple and minimally invasive technique, the outcome from our study indicates that it is not advisable for treatment of complete obstruction of the lacrimal drainage system. Balloon dilatation may prove suitable for the treatment of patients with partial obstruction below the level of the lacrimal sac, especially in those who are poor candidates for surgery, or who do not wish to undertake dacryocystorhinostomy. Even in the partial obstruction group the success rate was only $50 \%$, so that further modification to the technique and controlled studies are likely to be required before it could be recommended for general use. (Br F Ophthalmol 1999;83:692-696)

Uludag University, Department of Ophthalmology, Bursa, Turkey

B Yazici

H Erturk

Correspondence to: Zeynep Yazici, MD, Department of Radiology, Uludag University, School of Medicine, Gorukle Campus, 16059, Gorukle, Bursa, Turkey.

Accepted for publication 10 December 1998 sac and the nasal cavity to bypass the obstruction such as dacryocystorhinostomy (DCR). Dacryocystorhinostomy has a high success rate but it is an incisional surgical procedure. ${ }^{2}$ It may require general anaesthesia for the comfort of both the patient and the physician. Probing and silicone intubation are usually the treatment choice for the obstructive epiphora in children. The effectiveness of these methods, however, is quite limited in adults, probably because of the differences in the underlying cause of the obstruction. ${ }^{3}{ }^{4}$ The obstruction is usually caused by fibrosis as a result of idiopathic inflammation in adults, and imperforated Hasner's valve in children. Balloon dacryocystoplasty (BD) is a new method based on the recanalisation of the occluded lacrimal system. ${ }^{5}$ In this study, we investigated the efficacy of $\mathrm{BD}$ in 26 adult patients with obstructive epiphora.

\section{Materials and methods}

Between January 1995 and May 1997, BD was performed in 16 patients with complete obstruction and 10 patients with partial obstruction of the LDS (20 women and six men; age range 14-70 years; mean age 41 years). The average interval between the onset of symptomatic epiphora and the balloon dilatation was 34 months, with a range of 4 months to 15 years. Obstruction was idiopathic in all cases. All patients complained of moderate to severe epiphora. Each patient had an ophthalmic evaluation before the procedure to rule out an acute inflammatory process, and underwent digital subtraction dacryocystography (DCG) to determine the site and type of the obstruction. Patients with active dacryocystitis and canalicular obstruction were excluded from the study.

Local infiltration anaesthesia of the medial canthus with $2 \%$ lignocaine, topical conjunctival anaesthesia with $0.4 \%$ benoxinate hydrochloride, and nasal anaesthesia with $2 \%$ lignocaine and $0.5 \%$ phenylephrine spray were employed in the first eight patients. The remainder did not receive infiltration anaesthesia of the medial canthus. Following anaesthesia, in six patients a lacrimal probe of a lacrimal intubation set (Lacrimal intubation set 5010, Visitec, Sarasota, FL, USA) was introduced via the superior punctum and advanced through the obstruction site under fluoroscopic control. Then, a floppy tipped guide wire was advanced from the superior punctum into the inferior meatus, where a bayonet-shaped nasal forceps was used to catch the tip of the guide wire and was extended out of the nose. In the next 20 patients, neither lacrimal probe nor nasal forceps was used. Following the insertion of soft tip of the 0.014 or 0.018 inch guide wire (either straight or angled tipped: Ultra-select 

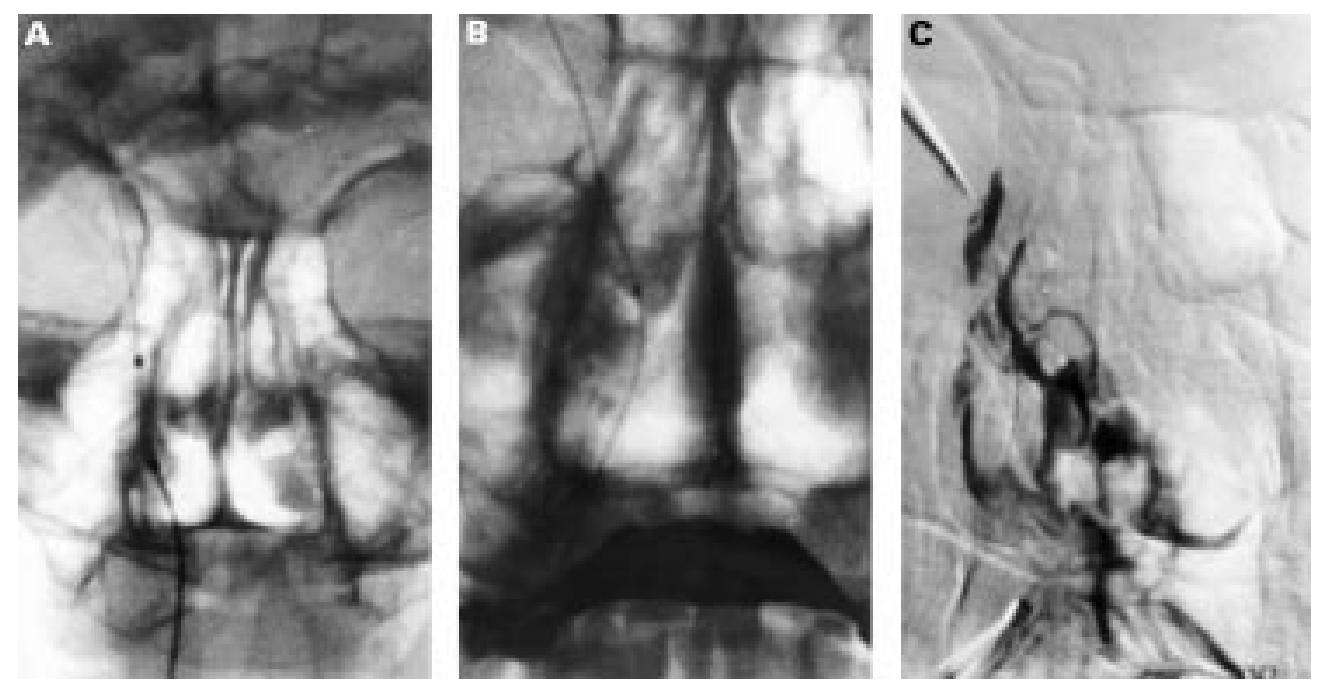

Figure 1 False passage creation. (A) Correct position of the guide wire. (B) The line of the guide wire is rather medial than usual trace. (C) Dacryocystogram obtained after balloon dacryocystoplasty shows that contrast medium is passing to the nasal cavity through the false passage.

nitinol guide wire, Microvena Corp, Minnesota, USA or Flexfinder, Flexmedics Corp, Minnesota, USA) via the superior punctum proximal to the obstructed segment, a 24 or 22 gauge plastic arterial sheath was introduced over the guide wire. Then, both the guide wire and the sheath were gently manipulated while trying to pass the guide wire through the obstruction site. This procedure was performed under fluoroscopy in lateral projection to see the trace of the guide wire through the LDS. After the tip of the guide wire was manipulated out of the nose, the sheath was removed superiorly from the canaliculus. A catheter with a $3 \mathrm{~mm}$ in diameter and $2 \mathrm{~cm}$ long balloon (Sure pass PTCA catheter, NuMed Canada Inc, Ontario, Canada or Dacryocystic duct dilation set, William Cook Europe A/S, Bjaevershov, Denmark) was introduced retrogradely over the guide wire and placed at the level of the obstruction under fluoroscopic control. In order to prevent damage to the canaliculi, special attention was paid not to place it higher than the sac. The balloon was inflated three to four times for 1 minute using a balloon inflation device. A radiograph was taken during balloon inflation to ensure the correct positioning of the balloon. By leaving the wire in place, the balloon catheter was removed inferiorly. DCG was performed by injecting the contrast medium via the inferior punctum to verify passage. The dilatation procedure was repeated when DCG revealed that the dilatation of the stenotic segment was not sufficient. Following the final dilatation, DCG was repeated to verify the success of the procedure. After completion of the intervention, the balloon was removed inferiorly and the wire superiorly. The LDS was irrigated with sterile saline. Topical antibiotic and steroid eye drops were administered postoperatively for 1 week in addition to oral antibiotic and antiinflammatory therapy for 5 days. On the following day, canalicular irrigation was performed to dislodge a possible clot. Within the first month after the procedure, canalicular irrigation was repeated weekly to determine the patency of the LDS. The patients were contacted every 2 months to obtain follow up information; patients having post-treatment epiphora underwent DCG.

\section{Results}

The follow up period ranged from 8 to 37 months (mean 14 months). Obstruction was at the lacrimal sac in five patients, the junction between the lacrimal sac and nasolacrimal duct in 14, in the nasolacrimal duct in five, and the diffuse stricture in two. Sixteen patients had complete obstruction and 10 had partial obstruction.

In four patients who had complete obstruction (one junctional and three in the lacrimal sac), BD could not be technically achieved. The failure in three patients was due to the inability to pass the guide wire through the obstruction site, and in one patient it was due to the creation of a false passage by the lacrimal probe (Fig 1). The technique was successful in 22 obstructed lacrimal ducts, consisting of 12 complete and 10 partial obstructions with a technical success rate of $85 \%$. The patients who did not receive medial canthal anaesthesia tolerated the operation well. No major complication was seen. The average time to perform the procedure was 47 minutes; the range was between 35 and 68 minutes.

Reobstruction occurred in 13 patients (eight complete obstruction and five partial obstruction). In seven of these 13 patients, reobstruction was observed within 2 months following the operation. Reobstruction occurred in five of six patients in whom a lacrimal probe was used to pass the obstruction site. No patency could be achieved in any complete obstruction located at the lacrimal sac. In one patient with severe partial obstruction, the type of the obstruction became complete after BD. During the follow up period, LDS remained patent after BD in $50 \%$ of the patients with partial obstruction and in $25 \%$ of those with complete obstruction (Figs 2 and 3). The overall success rate was $35 \%$ (Table 1 ). 

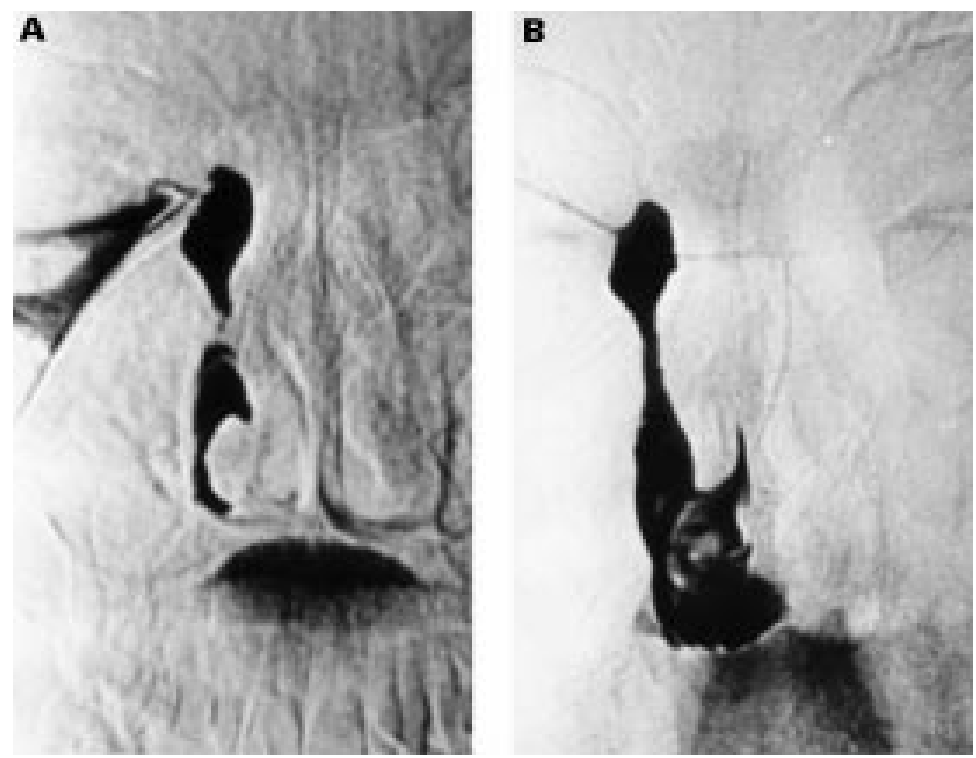

Figure 2 (A) Dacryocystogram shows partial passage of contrast medium through the partially obstructed nasolacrimal duct. (B) Dacryocystogram obtained after balloon dacryocystoplasty demonstrates resolution of the obstructed segment.
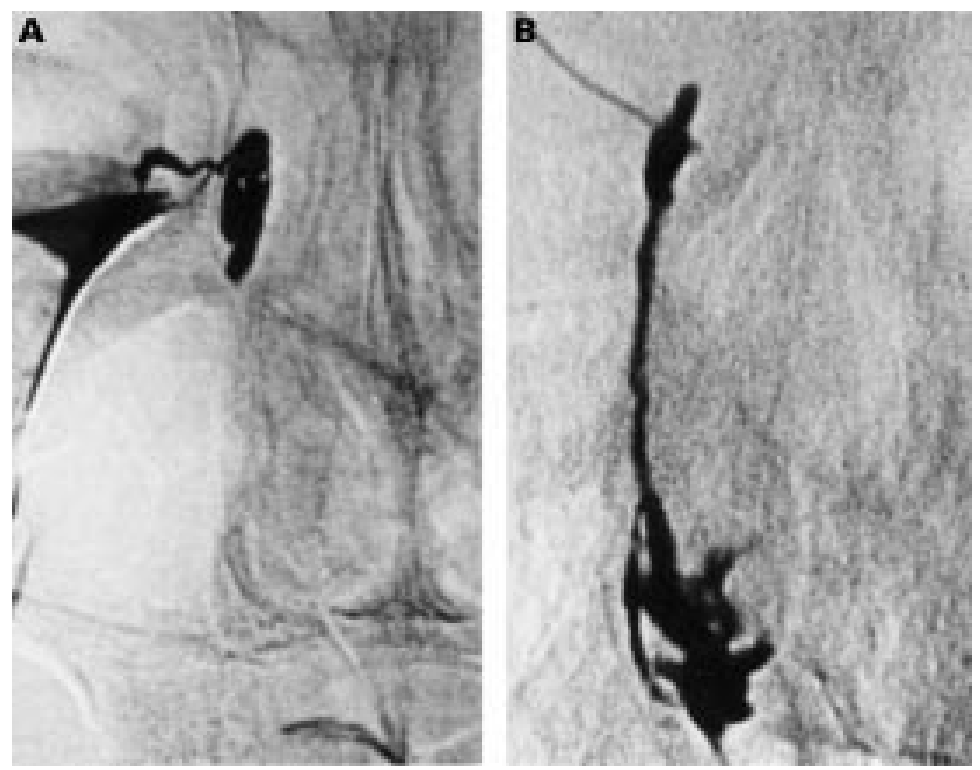

Figure 3 (A) Dacryocystogram shows the complete obstruction at the junction between lacrimal sac and nasolacrimal duct. (B) Dacryocystogram following balloon dacryocystoplasty reveals complete resolution of the obstruction site.

Table 1 Success versus obstruction site

\begin{tabular}{llll}
\hline Obstruction site & $\begin{array}{l}\text { Complete obstruction } \\
\text { (success/total) }\end{array}$ & $\begin{array}{l}\text { Partial obstruction } \\
\text { (success/total) }\end{array}$ & $\begin{array}{l}\text { Total } \\
\text { (success/total) }\end{array}$ \\
\hline Lacrimal sac & $0 / 5$ & - & $0 / 5(0 \%)$ \\
Junction & $3 / 9$ & $2 / 5$ & $5 / 14(36 \%)$ \\
Nasolacrimal duct & $1 / 2$ & $2 / 3$ & $3 / 5(60 \%)$ \\
Diffuse stricture & - & $1 / 2$ & $1 / 2(50 \%)$ \\
Total (n=26) & $4 / 16(25 \%)$ & $5 / 10(50 \%)$ & $9 / 26(35 \%)$ \\
\hline
\end{tabular}

$\star$ Junction refers to the junction between the nasolacrimal sac and duct.

\section{Discussion}

Although the success rate of external DCR is high, it is an invasive treatment of obstructive epiphora. In the past decade, endonasal endoscopic DCR, BD, and non-surgical treatment with stents have been proposed as an alternative to DCR..$^{5-7}$

The major technical difficulty of $\mathrm{BD}$ is to pass the soft tipped guide wire against an ana- tomical obstruction. ${ }^{8-11}$ While it is difficult to pass the obstruction with a soft tipped guide wire, a hard tipped guide wire tends to create a false passage and is more traumatic. ${ }^{12}$ Therefore the soft tipped guide wire has been widely used. ${ }^{9}{ }^{12-14}$ Robinson et $a l^{10}$ and Ilgit et $a l^{9}$ passed the obstruction site with a 21 gauge curved lacrimal cannula and a 20 gauge soft plastic arterial sheath supported intraluminally with a guiding lacrimal probe, respectively. We used the lacrimal probe to pass the obstruction site in our first six patients. However, a false passage was created in one of these patients. When the films obtained for this case were compared with others, we realised that the line of the guide wire was rather more medial than the usual trace. McCullough reported a similar event. $^{11}$ We think that the inappropriate passage of the wire was probably due to the perforation of the thin lacrimal bone by the probe and the advancement of the guide wire through this opening. The thickness of the lacrimal bone at the lacrimal sac fossa is mostly less than $100 \mu \mathrm{m}$, so that it can be easily penetrated with most surgical instruments. ${ }^{15}$ In the literature, the creation of a false passage has been reported to be through the submucosa or periosteum..$^{8-11}{ }^{13}$ The lacrimal probe was not used in the remaining patients in order to reduce the traumatic effects of the procedure. With the flexible guide wire, no false passage was created. The introduction of the guide wire through a plastic arterial sheath seems to facilitate the manipulation. McCullough modified the guide wire by reducing the length of the floppy tip for the same purpose. ${ }^{11}$

The difficulty in bringing forward the guide wire through the external nare can be overcome by using a haemostat under nasal endoscopic guidance or a hook. ${ }^{11}{ }^{13}$ In our experience, to direct the guide wire anteriorly with an angled tip was easy under fluoroscopic monitoring in the lateral projection. While it was easier to advance the guide wire through the obstruction site with the straight tip, the angled tip facilitated the advancement of the guide wire through the nare. Therefore, in some patients we first used a straight tipped guide wire to pass the obstruction, and after creating a track we replaced it with an angled tipped guide wire.

Munk et al were the first to perform retrograde balloon dilatation in adults with obstructive epiphora. ${ }^{5}$ There are several reports on BD with a significant difference in the success rates, varying from $23 \%$ to $89 \% .^{5} 8^{10-16}$ Janssen et al stated that the differences in results could be explained by differences in patient selection and different dacryocystoplasty techniques. ${ }^{14}$ However, on reviewing the literature, we think that it is hard to find a satisfactory explanation for the wide range in the success rates. Munk et al and McCullough included 18 patients (89\% partial) and 22 patients $(100 \%$ partial), respectively, with postsaccal obstructions in their studies. ${ }^{511}$ Munk et al and McCullough used the same sized balloon. Although the patient selection and the size of the balloon were similar in both studies, the durations of balloon inflation were 
different. Munk et al and McCullough inflated the balloon two or three times for 20-30 seconds each time and twice for 5 minutes each, respectively. Their overall success rates were $72 \%$ (Munk et al) and $68 \%$ (McCullough). These results show that the difference in the duration of balloon inflation may not affect the success rates significantly.

Lee et al included 20 patients with partial obstruction at the junction and nasolacrimal duct. ${ }^{13}$ Ilgit et al had 14 patients with partial obstruction at the nasolacrimal duct. ${ }^{9}$ They both inflated the $4 \mathrm{~mm}$ diameter balloon for at least 5 minutes. The overall success rates for partial obstruction in these two reports were $25 \%$ and $100 \%$, respectively. Although there was not a significant difference in the techniques and the type and the site of the obstruction, there was a remarkable difference between the results. Ilgit et al concluded that their higher overall success rate compared with that reported by Lee et al might be attributed to the different average intervals between the epiphora and the BD. ${ }^{9}$ However, Janssen et al did not find any statistically significant difference in the success rate between patients with epiphora for 1 year or less and those with epiphora for more than 1 year. ${ }^{14}$

According to the results of the previous studies, it seems that the treatment of epiphora by $\mathrm{BD}$ is more successful for partial obstructions than for complete obstructions, and in nasolacrimal duct obstructions than in obstructions of the lacrimal sac or the canaliculi. ${ }^{1011} 14^{16}$ Our results tend to confirm these observations. In our study, the results of $\mathrm{BD}$ in partial obstruction were better than those in complete obstruction $(50 \%$ versus $25 \%)$. We obtained the highest patency rate $(60 \%)$ in the patients with nasolacrimal duct obstruction. But the relatively small number of patients in this group as opposed to the other groups does not allow us to make further conclusions in terms of the site of the obstruction. The success rate was significantly lower in patients in whom a lacrimal probe was used to pass the obstruction site. It is obvious that the traumatic effect of the procedure to the LDS is an essential factor in the failure of BD.

In their widely quoted clinicopathological study, Linberg and McCormick reported that the reason of primary acquired nasolacrimal duct obstruction in the early phase is active chronic inflammation along the entire length of the narrowed nasolacrimal duct, where fibrous obliteration eventuates in the late phase. ${ }^{1}$ Although $\mathrm{BD}$ resolves the obstruction, it does not remedy the primary inflammatory process, which is likely to affect the recurrence of the obstruction. As the balloon is inflated with pressure against the stenotic area, the mucosa of the nasolacrimal duct gets crushed which probably causes development of inflammatory reaction and haemorrhage, eventually increasing the likelihood of the development of the reobstruction. For this reason, the use of topical or systemic anti-inflammatory agents to reduce the early inflammatory reaction and saline irrigation following the operation to dislodge the clot formation have been suggested to prevent the development of reobstruction. ${ }^{814}$ However, this treatment regimen may not be successful in all cases, as demonstrated by our study.

A potential criticism of the present study may be that the radiation dose to the eye during BD was not measured. Indeed, interventional radiological procedures take much more time than do routine diagnostic $x$ ray examinations; therefore, there is increased risk of radiation exposure to the patients and the personnel involved. The clinical threshold for the development of radiation induced cataract in acute exposure is 5 Gy (2-10 Gy). ${ }^{17}$ The radiation dose to the head during the interventional neuroradiological procedures is much smaller than the threshold level for the eye. ${ }^{17} 18$ Since BD generally takes less time than neuroangiointerventional procedures, we can assume that the radiation dose to the eye did not reach the critical level in the present study.

The success rates reported in the literature for simple probing in adults are almost identical to the success rate found in our study. ${ }^{19}$ It would be unlikely to produce a persistent patency with probing or $\mathrm{BD}$ in a long standing obstruction when a dense fibrous tissue has already developed. Balloon dacryocystoplasty with subsequent silicone intubation or stent placement seems to ensure the patency of the system. ${ }^{20}{ }^{21}$ Our overall success rate with $\mathrm{BD}$ is low compared with DCR; however, BD is less traumatic, easier to perform, and could be more promising in some cases. Further investigations may improve the efficacy of this method.

1 Linberg JV, McCormick SA. Primary acquired nasolacrimal duct obstruction: a clinicopathologic report and biopsy technique. Ophthalmology 1986;93:1055-63.

2 Tarbet KJ, Custer PL. External dacryocystorhinostomy: surgical success, patient satisfaction, economic cost. Ophthalmology 1995;102:1065-70.

3 Bell TAG. An investigation into the efficacy of probing the nasolacrimal duct as a treatment for epiphora in adults. Trans Ophthalmol Soc UK 1986;105:494-7.

4 Becker BB, Berry FD, Koller H. Balloon catheter dilatation for treatment of congenital nasolacrimal duct obstruction. Am f Ophthalmol 1996;121:304-9.

5 Munk PL, Lin DTC, Morris DC. Epiphora: treatment by means of dacryocystoplasty with balloon dilation of the nasolacrimal drainage apparatus. Radiology 1990;177:68790 .

6 Shun-shin GA, Thurairajan G. External dacryocystorhinostomy — an end of an era? Br f Ophthalmol 1997;81:71617.

7 Song HY, Ahn HS, Park CK, et al. Complete obstruction of the nasolacrimal system. Part II. Treatment with lacrimal metallic stents. Radiology 1993;186:372-6.

8 Song HY, Ahn HS, Park CK, et al. Complete obstruction of the nasolacrimal system. Part I. Treatment with balloon dilation. Radiology 1993;186:367-71.

9 Ilgit ET, Yuksel D, Unal M, et al. Transluminal balloon dilatation of the lacrimal drainage system for the treatment of epiphora. Am f Roentgen 1995;165:1517-24.

10 Robinson R, Turner N, Brettle P, et al. The treatment of epiphora with balloon dacryocystoplasty. Eye 1993;7:68790.

11 McCullough KM. Naso-lacrimal duct balloon dilatation. Clin Radiol 1994;49:787-90.

12 Berkefeld J, Kirchner J, Muller HM, et al. Balloon dacryocystoplasty: indications and contraindications. Radiology 1997;205:785-90.

13 Lee JM, Song HY, Han YM, et al. Balloon dacryocystoplasty: results in the treatment of complete and partial obstructions of the nasolacrimal system. Radiology 1994;192:503-8.

14 Janssen AG, Mansour K, Bos JJ. Obstructed nasolacrimal duct system in epiphora: long-term results of dacryocystoplasty by means of balloon dilation. Radiology 1997;205: plasty 6 .

15 Hartikainen J, Aho HJ, Seppa H, et al. Lacrimal bone thickness at the lacrimal sac fossa. Ophthalmic Surg Lasers 1996; 27:679-84. 
16 Kumar E. Technical note: non-surgical treatment of epiphora by balloon dacryocystoplasty-the technique. $\mathrm{Br} \mathcal{F}$

17 Kuwayama N, Takaku A, Endo S, et al. Radiation exposure in endovascular surgery of the head and neck. Am F Neuroradiol 1994;15:1801-8.

18 Bergeron P, Carrier R, Roy D, et al. Radiation doses to patients in neurointerventional procedures. Am $\mathcal{F}$ Neuroradiol 1994;15:1809-12.
19 Bell TAG. An investigation into the efficacy of probing the nasolacrimal duct as a treatment for epiphora in adults. Ophalmol Soc UK 1986;105:494-7.

20 Steinkogler FJ, Huber E, Kuchar A, et al. Retrograde dilation of postsaccal lacrimal stenosis. Ann Otol Rhinol Laryngol 1994;103:110-14 .

21 Song HY, Jin YH, Kim JH, et al. Nonsurgical placement of a nasolacrimal polyurethane stent: long term effectiveness. Radiology 1996;200:759-63. 